\title{
PROJEKTPLANUNG BEI UNSCHARFEN DATEN. INFORMATIONSVERARBEITUNG UND EINBEZIEHUNG VON BESCHLEUNIGUNGSKOSTEN
}

\section{Heinrich J. Rommelfanger, J.W. Goethe-Universität Frankfurt a.M.}

Netzpläne stellen ein geeignetes Instrumentarium zur Projektplanung dar, wie die umfangreiche Literatur $z u$ diesem Themenkomplex und zahlreiche Realisierungen in der Praxis zeigen. Dies gilt aber nur unter der Annahme, daß die Ablaufstruktur bekannt ist und die Zeitdaten der einzelnen Teilprojekte exakt angegeben werden können. Da insbesondere die Dauer vieler Vorgänge als zukunftsorientierte Größe zum Planungszeitpunkt nur größenordnungsmäßig festgelegt werden kann, wird in neueren Arbeiten vorgeschlagen, Netzpläne mit Fuzzy-Daten als Planungsgrundlage $\mathrm{zu}$ verwenden. In /1/ wird gezeigt, daß sich die Konzepte der MPM-Netzplantechnik auch auf unscharfe Netzpläne übertragen lassen, wenn man beachtet, daß im Gegensatz zum reellen Zahlenraum bei Fuzzy Zahlen die erweiterte Subtraktion i. a. nicht die Umkehr der erweiterten Addition darstellt.

In diesem Beitrag wird untersucht, ob durch Verwendung unscharfer Netzpläne die Qualität der Projektplanung gesteigert werden kann und ob die damit verbundene zielgerichtete Informationsbeschaffung zu einer Senkung der Planungskosten und der Planungszeit führt. Weiterhin wird analysiert, wie Beschleunigungskosten in Netzpläne mit FuzzyDaten integriert werden können. Ein Modell zur Berechnung des kostenminimalen Maximalflusses wird vorgestellt und an einem Zahlenbeispiel erläutert.

/1/ Rommelfanger, H.: Netzplantechnik mit Fuzzy-Daten. Operations Research Proceedings _1992, Springer-Verlag Berlin Heidelberg 1993 